\title{
Appetitive Pavlovian goal-tracking memories reconsolidate only under specific conditions
}

\author{
Amy C. Reichelt ${ }^{1}$ and Jonathan L.C. Lee \\ School of Psychology, University of Birmingham, Edgbaston, Birmingham B15 2TT, United Kingdom
}

\begin{abstract}
Despite extensive evidence that appetitive memories undergo reconsolidation, two notable failures to observe reconsolidation have been reported: instrumental responding and goal-tracking. However, these studies do not provide conclusive evidence for a lack of memory reconsolidation due to the numerous boundary conditions that dictate whether a memory will undergo reconsolidation. In this study we sought to reexamine reconsolidation in an appetitive, Pavlovian conditioned approach procedure and the behavioral boundary conditions within which memories are destabilized and reconsolidated. This study demonstrated that a Pavlovian goal-tracking memory, previously thought to be resistant to destabilization, will undergo memory reconsolidation under discrete conditions that favor reconsolidation as opposed to extinction, and that this is dependent on the amount of training rats received. With restricted training, systemic administration of MK801 impaired memory extinction. In contrast, with more extended training, MK-801 administration impaired memory reconsolidation. We also demonstrate that behavioral boundary conditions that exist for appetitive memory reconsolidation are much more complex than simple parametric calculations. Moreover, extinction per se is not a boundary on reconsolidation, in that MK-801 also has no behavioral effect under some conditions.
\end{abstract}

Memories are dynamic, rather than static, in nature and can be updated to maintain their relevance in day-to-day life. Reactivation of a memory through reexposure to associated stimuli results in its destabilization in which the memory can become susceptible to changes and also can be disrupted by amnestic agents (Alberini 2005). Restabilization of this labile memory allows the return to a stable, persistent state by reconsolidation, and a disruption of this process leads to amnesia (Lee 2008).

There is extensive evidence now that appetitive memories undergo reconsolidation, the process by which memories are restabilized following their reactivation. This has been demonstrated in a number of experimental paradigms using natural (Diergaarde et al. 2006; Lee and Everitt 2008a), alcohol (Wouda et al. 2010; Milton et al. 2012), and addictive drug rewards (Lee et al. 2005, 2006a; Milton et al. 2008a,b). However, there are two notable failures to observed memory reconsolidation in appetitive settings: instrumental responding (Hernandez and Kelley 2004) and Pavlovian goal-tracking (Blaiss and Janak 2007). Nevertheless, these individual negative findings are not conclusive evidence for a lack of memory reconsolidation, especially given the numerous "boundary conditions" on the reconsolidation process (for review, see Nader and Hardt 2009; Hardt et al. 2010; Finnie and Nader 2012).

Without clear and systematic manipulations exploring the boundaries within which reconsolidation occurs it is difficult to assess whether the failure to observe reconsolidation in a given setting is due to behavioral parameters of memory retrieval being inappropriate for the induction of reconsolidation or because the underlying memory genuinely does not undergo reconsolidation under any circumstances. There are actually numerous observations that, within an experimental paradigm, memories do undergo reconsolidation, but not always. This has led to the description of a number of boundary conditions on memory reconsolidation that determine whether or not memory retrieval leads to memory

'Corresponding author

E-mail a.c.reichelt@bham.ac.uk

Article is online at http://www.learnmem.org/cgi/doi/10.1101/lm.027482.112. reconsolidation. We have recently argued that the different boundary conditions might be unified under the notion that memory retrieval must result in memory modification in order to trigger memory reconsolidation (Lee 2009). Conversely, the hypothesis that reconsolidation mediates memory updating may suggest that reconsolidation should be a universal property of memory processing (Lee 2009). As such, the universality of reconsolidation remains an important question.

One of the more fully explored boundary conditions, at least in Pavlovian memory settings, is that of the balance between the strength of initial memory acquisition and the extent of nonreinforced CS exposure at memory retrieval. The standard method of memory reactivation is a short session in which cues are presented without the US. Typically, a short reactivation procedure is likely to induce reconsolidation in memories that are weak and relatively new as opposed to strong, established memories (Nader and Einarsson 2010). However this session may also induce extinction learning in which a new CS-no US memory is formed and conditioned responding to the CS is inhibited by the new association (Konorski 1967; Robbins 1990; Rescorla 2001). Thus, retrieval of the CS-US association during reactivation may initiate either strengthening of the memory via reconsolidation (Inda et al. 2011) or weakening of memory expression, demonstrating that extinction and reconsolidation are functionally distinct processes (Suzuki et al. 2004; Tronson and Taylor 2007). Studies have demonstrated that when conditioning is kept constant, the duration of reactivation is crucial to whether a memory reconsolidates or extinguishes (Pedreira and Maldonado 2003; Lee et al. 2006b). Alternatively, the strength of initial training determines whether a retrieval session of fixed duration engages reconsolidation or extinction processes (Eisenberg et al. 2003). Thus, the strength of a memory is an important factor with respect to whether a reactivation session induces reconsolidation or extinction.

Less is known about the boundary conditions that may exist with respect to the reconsolidation of appetitive memories. Currently, no evidence of a strength boundary condition exists in a cocaine-seeking setting, indicating that even extensively conditioned drug memories can undergo reconsolidation (Lee et al. 
2006a). This suggests that, although they are strong and longlasting, established drug memories are susceptible to reconsolidation disruption. Paradoxically, it was noted that cocaine memories may be more susceptible to reconsolidation than natural reward memories (Milton et al. 2008b). A more persistent reduction in cocaine-associated behaviors was evoked in comparison to a sucrose-seeking behavior following propranolol administration in conjunction with memory reactivation (Milton et al. 2008b). Moreover, cue-cocaine memories were more easily destabilized when cues were presented in a different manner to that experienced during learning than were cue-sucrose memories (Lee et al. 2006a; Lee and Everitt 2008b). Therefore, the lack of current observations of boundary conditions on addictive drug memory reconsolidation is not clearly predictive of patterns of results using natural rewards. Combined with the acknowledgment that the failure to observe reconsolidation in an instrumental memory may be due to the memory being too strong (Hernandez and Kelley 2004), current negative findings are not sufficient to conclude that reconsolidation does not occur in instrumental and Pavlovian goal-tracking settings.

Given the established principles of boundary conditions in aversive Pavlovian memory reconsolidation, we have focused in this study on appetitive Pavlovian goal-tracking, whereby presentations of a discrete cue promote a conditioned approach to the magazine port where rewards are delivered. The failure to observe reconsolidation in instrumental memory (Hernandez and Kelley 2004) and also in goal-tracking (Blaiss and Janak 2007) may be due to insufficient exploration into the boundary conditions that underpin reconsolidation. In the study conducted by Blaiss and Janak (2007) the possibility of strength of training as a boundary condition was explored by varying the number of days of training. However, post-reactivation anisomycin did not disrupt memory reconsolidation under any of the training conditions used. In contrast, there is some weak evidence that within a Pavlovian sign-tracking setting, in which a cue light indicated the availability of an appetitive reward, goal-tracking behavior was impaired when reconsolidation was disrupted (Lee and Everitt 2008a; Milton et al. 2012). These observations resulted from the amnestic effects of the systemic administration of the NMDA receptor antagonist MK-801. Therefore, given the importance of the question of whether or not reconsolidation is a universal property of memory processing, we have reexamined the vulnerability of goal-tracking memories to reconsolidation disruption in a pure Pavlovian conditioned approach setting.

\section{Experiment 1: Reexamination of goal-tracking memory reconsolidation}

\section{Results}

Rats were trained to respond to two discriminable auditory stimuli (click or tone, CS+ and CS-, 10 presentations of each per session) for 3,6 , or $12 \mathrm{~d}$. Following training, a short reactivation procedure took place in the reactivation groups, whereby rats were exposed to three nonreinforced presentations of each CS. The rats were administered MK-801 (0.1 mg/kg i.p.) or saline prior to the reactivation procedure. In the nonreactivation condition the rats were administered MK-801 or saline, but did not experience the reactivation procedure. Testing took place $24 \mathrm{~h}$ later with both CSs presented in extinction.

\section{Criterion for analysis}

Rats were excluded from the analysis if they failed to show adequate discrimination between the $\mathrm{CS}+$ and $\mathrm{CS}-$ on the final day of training. The criterion used was whether the suppression ratios calculated on the final day of training were $\mathrm{CS}+>0.50$ and $\mathrm{CS}+$ $>\mathrm{CS}-$. This ensured that rats elevated responding during the CS presentations compared to during the 30 -sec period preceding the CS presentation (PreCS), and rats biased responding to the rewarded stimulus.

\section{Experiment la: 3-d training}

A total of 52 rats were used in this experiment, of which five were excluded from the reactivation experiment (total, $n=23$; saline, $n=12$; MK-801, $n=11$ ) and four from the nonreactivation experiment (total, $n=20$; saline, $n=10$; MK-801, $n=10$ ). Rats were excluded on the basis of failing to acquire the discriminations within the 3-d training procedure in accordance to the criterion for analysis detailed previously.

\section{Reactivated rats}

Acquisition of Pavlovian discriminations. Figure 1A shows the mean rate of magazine entry responding during the course of acquisition. Inspection of this figure shows that rats displayed discriminated responding to the CS+ over the CS-. A within-subjects ANOVA with a between-subjects factor of the drug (MK-801, saline) and within-subjects factors of the training session (1-3) and the CS $(\mathrm{CS}+, \mathrm{CS}-)$ revealed a significant main effect of the session
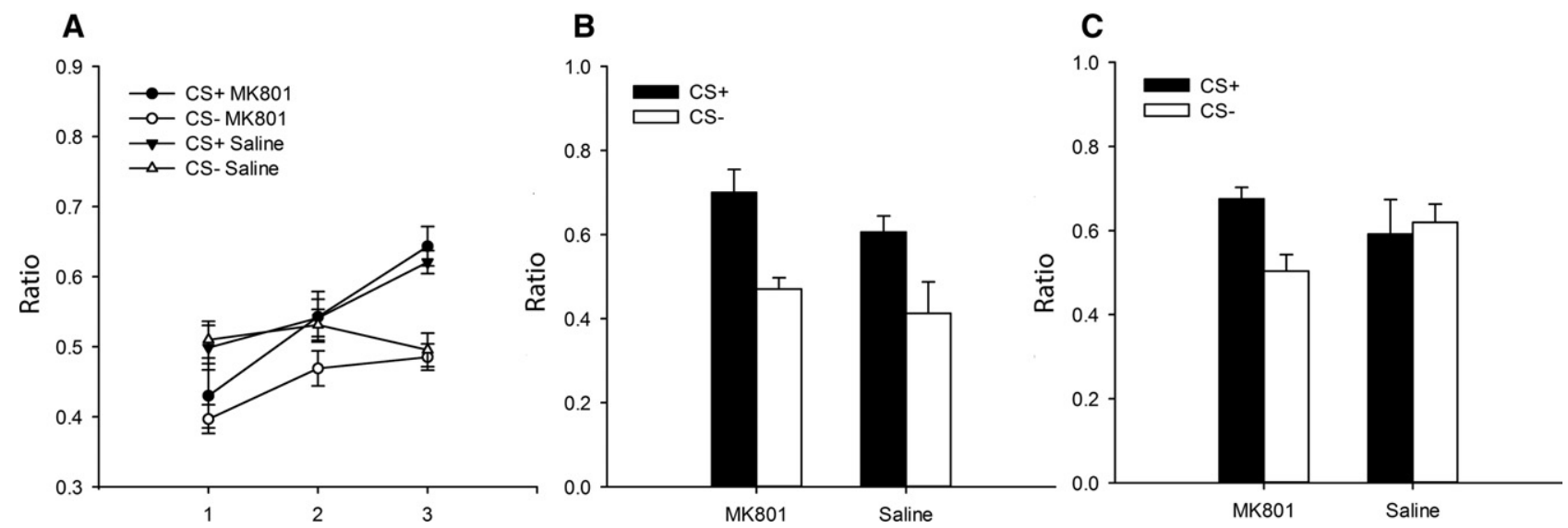

Figure 1. (A) Acquisition of individual auditory (CS,$+ \mathrm{CS}-$ ) discriminations across the 3 -d training protocol (saline, $n=12 ; \mathrm{MK}-801, n=11)$. (B) Responding to three presentations each of the nonreinforced CS + and CS- during the brief reactivation session. (C) Probe test responding to 10 presentations each of the CS + and CS- in extinction. Error bars represent \pm 1 SEM. All data are shown as ratios calculated as magazine responding during $\mathrm{CS} /(\mathrm{CS}+$ PreCS). 
$\left(F_{(2,42)}=14.071, P<0.001\right)$, a significant main effect of the drug $\left(F_{(1,21)}=4.74, P<0.05\right)$, and a significant main effect of the CS $\left(F_{(1,21)}=23.372, P<0.001\right)$. A significant interaction between the session $\times$ CS was observed $\left(F_{(2,42)}=5.82, P<0.01\right)$. No further interactions were significant $\left(F^{\prime} \mathrm{s}<3.187\right)$. Simple effects analysis of the session $\times$ CS interaction revealed significant effects of the CS in session $3\left(F_{(1,21)}=74.412, P<0.001\right)$, but not in sessions 1 and 2 ( $\left.F^{\prime} \mathrm{s}<2.263\right)$. Significant effects of the session were observed during CS + presentations $\left(F_{(2,20)}=10.722, P<\right.$ $0.01)$, but not during $C S$ - ones $\left(F_{(2,20)}=2.161, P=0.141\right)$.

Reactivation. Figure 1B shows the mean rate of magazine entry responding during the reactivation CS presentations in rats administered saline or MK-801 prior to the reactivation procedure. Inspection of this figure shows that rats administered both saline and MK-801 maintained a Pavlovian conditioned approach response to the CS+ but not to the CS-. A mixed ANOVA with within factors of the CS (CS +, CS-) and a between-subjects factor of the drug (MK-801, saline) revealed a significant main effect of the CS $\left(F_{(1,21)}=11.300, P<0.01\right)$ and no significant main effect of the drug $\left(F_{(1,21)}=3.569, P=0.073\right)$. No significant interaction between the drug $\times$ CS was observed $\left(F_{(1,21)}<1\right)$.

Test performance. Figure 1C shows the mean rate of magazine entry responding during the extinction test trial in rats administered saline or MK-801 prior to the reactivation procedure. Inspection of this figure illustrates that rats administered MK-801 maintained a Pavlovian conditioned approach response to the CS+ but not to the CS-. In contrast, saline-injected rats showed no discrimination between the CS + and $\mathrm{CS}-$. A mixed ANOVA with within factors of the CS (CS +, CS-) and a between-subjects factor of the drug (MK-801, saline) revealed no significant main effect of the CS $\left(F_{(1,21)}=2.709, P=0.115\right)$ and no significant main effect of the drug $\left(F_{(1,21)}<1\right)$. Importantly, a significant interaction between the drug $\times$ CS was observed $\left(F_{(1,21)}=5.241\right.$, $P<0.05)$. Simple effects analysis of the drug $\times$ CS interaction revealed a significant effect of the CS in rats administered MK-801 $\left(F_{(1,21)}=7.420, P<0.05\right)$, but not in rats administered saline $\left(F_{(1,21)}<1\right)$. Thus, the reactivation procedure induced extinction in rats administered saline and MK-801 administration was effective in impairing extinction consolidation.

\section{Nonreactivated rats}

Acquisition of Pavlovian discriminations. Figure 2A shows the mean rate of magazine entry responding during the course of acquisition. Inspection of this figure shows that rats acquired a Pavlovian conditioned approach response to the $\mathrm{CS}+$ but not to the CS-. A within-subjects ANOVA with a between-subjects factor of the drug (MK-801, saline) and within factors of the training session (1-3) and CS $(\mathrm{CS}+, \mathrm{CS}-)$ revealed a significant main effect of the session $\left(F_{(2,36)}=7.42, P=\right.$ $0.01)$, a significant main effect of the CS $\left(F_{(1,18)}=39.886, P<0.001\right)$, and no significant main effect of the drug $(F<1)$. No significant interactions were observed $\left(F^{\prime} \mathrm{s}<2.176\right)$.

Test performance. Figure 2B shows the mean rate of magazine entry responding during the extinction test trial in rats administered saline or MK-801 prior to the reactivation procedure. Inspection of this figure shows that rats administered MK-801 or saline maintained a Pavlovian conditioned approach response to the $\mathrm{CS}+$ but not to the CS-. A mixed ANOVA with within factors of the CS (CS +, CS-) and a between-subjects factor of the drug (MK-801, saline) revealed a significant main effect of the CS $\left(F_{(1,18)}=9.084, P<0.01\right)$ and no significant main effect of the drug $\left(F_{(1,16)}<1\right)$. No significant interaction between the drug $\times$ CS was observed $\left(F_{(1,16)}<1\right)$. Thus, without the reactivation procedure, MK-801 had no effect on discriminative responding in rats.

\section{Experiment lb: 6-d training}

A total of 36 rats were used in this experiment. Two rats were excluded from the reactivated group (total, $n=20$; saline, $n=10$; MK-801, $n=10$ ) as they failed to acquire the discriminations to the criterion following the 6 -day training period and 16 rats were used as nonreactivated controls (saline, $n=8$; MK-801, $n=8$ ).

\section{Reactivated rats}

Acquisition of Pavlovian discriminations. Figure 3A shows the mean rate of magazine entry responding during the course of acquisition. Inspection of this figure shows that rats acquired a Pavlovian conditioned approach response to the CS+ but not to the CS-. A within-subjects ANOVA with a between-subjects factor of drug (MK-801, saline) and within factors of the training session (1-6) and the CS (CS +, CS-) revealed a significant main effect of the session $\left(F_{(5,80)}=16.859, P<0.001\right)$, a significant main effect of the CS $\left(F_{(1,16)}=40.727, P<0.001\right)$, and no significant effect of the drug $\left(F_{(1,16)}=1.161, P=0.297\right)$. A significant interaction between the session $\times$ CS was observed $\left(F_{(5,80)}=6.674, P<0.001\right)$ and no further interactions were significant $\left(F^{\prime} \mathrm{s}<0.782\right)$. Simple effects analysis of the session $\times$ CS interaction revealed significant effects of the CS in session 1 and from session four onward $\left(F_{(1,16)}\right.$ 's $\left.>5.504\right)$. Significant effects of the session were observed for magazine responding to both CS $+\left(F_{(5,12)}=23.022\right.$, $P<0.001)$ and CS $-\left(F_{(5,12)}=8.193, P<0.01\right)$.

Reactivation. Figure 3B shows the mean rate of magazine entry responding during the reactivation CS presentations in rats administered saline or MK-801 prior to the reactivation procedure. Inspection of this figure shows that rats administered
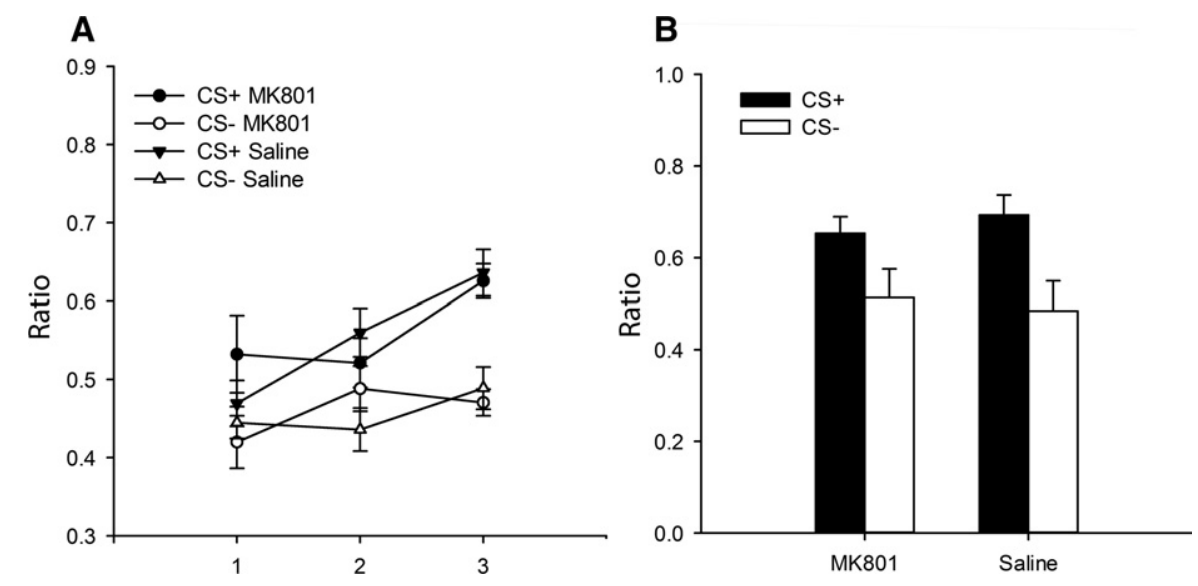

Figure 2. (A) Acquisition of individual auditory (CS +, CS-) discriminations across the 3-d training protocol (saline, $n=10 ;$ MK-801, $n=10$ ). (B) Probe test responding to 10 presentations each of the $\mathrm{CS}+$ and CS - in extinction. Error bars represent \pm 1 SEM. All data are shown as ratios calculated as magazine responding during $\mathrm{CS} /(\mathrm{CS}+\mathrm{PreCS})$. 

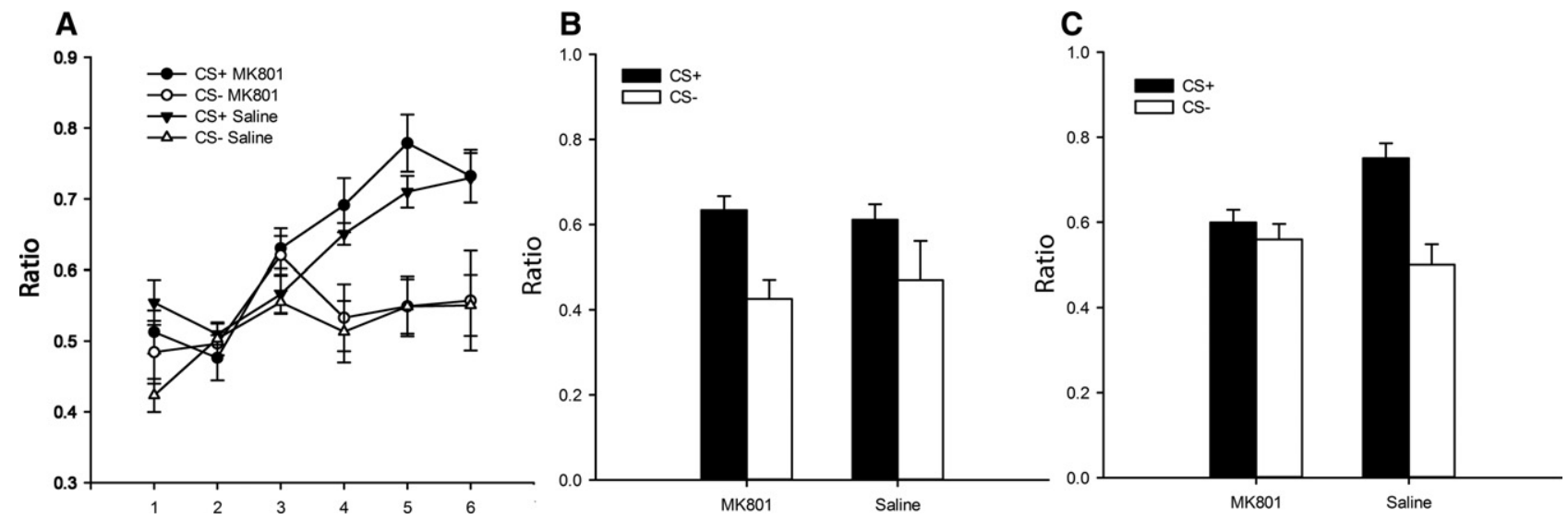

Figure 3. (A) Acquisition of individual auditory (CS,$+ \mathrm{CS}-$ ) discriminations across the 6-d training protocol (saline, $n=10 ; \mathrm{MK}-801, n=10)$. (B) Responding to three presentations each of the nonreinforced CS + and CS- during the brief reactivation session. (C) Probe test responding to 10 presentations each of the CS + and CS- in extinction. Error bars represent \pm 1 SEM. All data are shown as ratios calculated as magazine responding during $\mathrm{CS} /(\mathrm{CS}+$ PreCS).

both saline and MK-801 maintained a Pavlovian conditioned approach response to the CS+ but not to the CS-. A mixed ANOVA with within factors of the CS (CS,$+ \mathrm{CS}-)$ and a between-subjects factor of the drug (MK-801, saline) revealed a significant main effect of the CS $\left(F_{(1,18)}=8.778, P<0.01\right)$ and no significant main effect of the drug $\left(F_{(1,18)}<1\right)$. No significant interaction between the drug $\times$ CS was observed $\left(F_{(1,18)}<1\right)$.

Test performance. Figure 3C shows the mean rate of magazine entry responding during the extinction test trial in rats administered saline or MK-801 prior to the reactivation procedure. Inspection of this figure shows that rats administered saline maintained a Pavlovian conditioned approach response to the CS+ but not to the CS - ; however, rats administered MK-801 did not bias Pavlovian responding during the $\mathrm{CS}+$ presentations. A mixed ANOVA with within factors of the CS (CS +, CS-) and a between-subjects factor of the drug (MK-801, saline) revealed a significant main effect of the CS $\left(F_{(1,16)}=13.099, P<0.01\right)$ and no significant main effect of the drug $\left(F_{(1,16)}=1.679, P=\right.$ 0.213). Importantly, a significant interaction between the drug $\times$ CS was observed $\left(F_{(1,16)}=6.938, P<0.05\right)$. Simple effects analysis of the drug $\times$ CS interaction revealed a significant effect of the CS in rats administered prereactivation saline $\left(F_{(1,16)}=10.903\right.$, $P<0.01)$, but not in rats administered MK-801 $\left(F_{(1,16)}<1\right)$. Significant effects of the drug were observed during $\mathrm{CS}+$ presentations $\quad\left(F_{(1,16)}=19.552, \quad P<\right.$ $0.001)$ but not during CS- presentations $\left(F_{(1,16)}<1\right)$, thus indicating that MK-801 abolished discrimination between the $\mathrm{CS}+$ and $\mathrm{CS}-$ in rats that underwent the reactivation procedure.

\section{Nonreactivated rats}

Acquisition of Pavlovian discriminations. Figure 4A shows the mean rate of magazine entry responding during the course of acquisition. Inspection of this figure shows that rats acquired a Pavlovian conditioned approach response to the CS+ but not to the CS-. A within-subjects ANOVA with a between-subjects factor of the drug (MK-801, saline) and within-subjects factors of the training session (1-6) and the $\mathrm{CS}(\mathrm{CS}+, \mathrm{CS}-)$ revealed a significant main effect of the session $\left(F_{(5,70)}=10.283, P<0.001\right)$, no significant main effect of the drug $\left(F_{(1,14)}=1.591, P=0.228\right)$, and a significant main effect of the CS $\left(F_{(1,14)}=13.245, P<\right.$ 0.01). A significant interaction between the session $\times$ CS was observed $\left(F_{(5,70)}=3.372, P<0.01\right)$. Simple effects analysis of the session $\times$ CS interaction revealed significant effects of the CS from session 4 onward $\left(F_{(1,14)}\right.$ 's $\left.>4.755\right)$. Significant effects of the session were observed for both the CS $+\left(F_{(5,10)}=23.329, P<\right.$ $0.001)$ and the CS- $\left(F_{(5,10)}=5.919, \quad P<0.01\right)$ magazine responding.

Test performance. Figure 4B shows the mean rate of magazine entry responding during the extinction test trial in rats administered saline or MK-801, but that did not experience the reactivation procedure. Inspection of this figure shows that rats administered saline or MK-801 maintained a Pavlovian conditioned approach response to the $\mathrm{CS}+$ but not to the $\mathrm{CS}-$. A mixed ANOVA with within factors of the CS (CS +, CS-) and a betweensubjects factor of drug (MK-801, saline) revealed a significant main effect of the CS $\left(F_{(1,14)}=6.85, P<0.05\right)$ and no significant
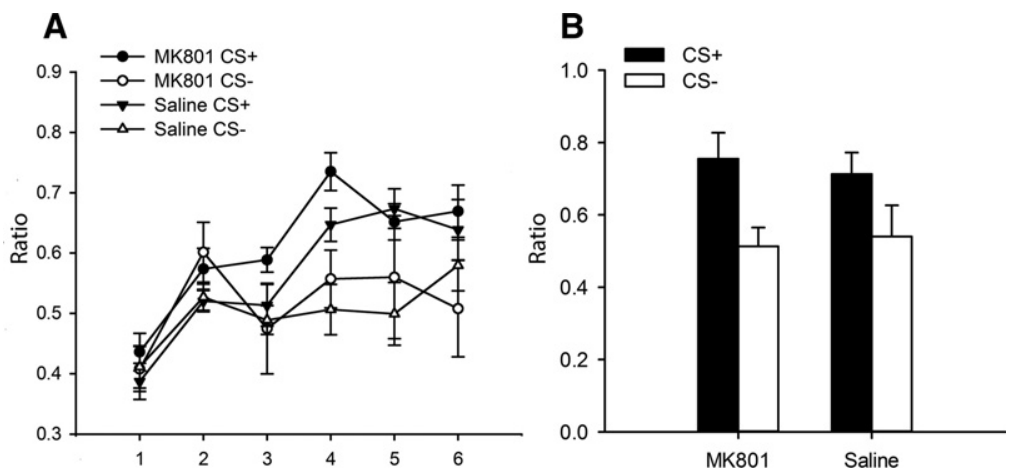

Figure 4. (A) Acquisition of individual auditory (CS + , CS-) discriminations across the 6-d training protocol (saline, $n=8$; MK-801, $n=8$ ). (B) Probe test responding to 10 presentations each of the $\mathrm{CS}+$ and CS - in extinction. Error bars represent \pm 1 SEM. All data are shown as ratios calculated as magazine responding during $\mathrm{CS} /(\mathrm{CS}+\mathrm{PreCS})$. 
main effect of the drug $\left(F_{(1,14)}<1\right)$. No significant interaction between the drug $\times$ CS was observed $\left(F_{(1,14)}<1\right)$. Thus, MK-801 did not abolish discrimination responding between the CS+ and CS- in rats that did not undergo the reactivation procedure.

\section{Experiment 1c: 12-d training}

A total of 16 rats were used in this experiment (saline, $n=8$; MK-801, $n=8$ ). All the rats acquired the discriminations to criterion.

\section{Acquisition of Pavlovian discriminations}

Figure 5A shows the mean rate of magazine entry responding during the course of acquisition. Inspection of this figure shows that the rats acquired a Pavlovian conditioned approach response to the $\mathrm{CS}+$ but not to the CS-. A within-subjects ANOVA with a between-subjects factor of the drug (MK-801, saline) and withinsubjects factors of the training session (1-12) and the CS (CS +, CS-) revealed a significant main effect of the session $\left(F_{(11,154)}=\right.$ 12.947, $P<0.001)$, a significant main effect of the CS $\left(F_{(1,14)}=\right.$ 48.397, $P<0.001$ ), and no significant main effect of the drug $(F<1)$. A significant interaction between the session $\times$ CS was observed $\left(F_{(11,165)}=2.821, P<0.01\right)$, and no further interactions were significant $\left(F^{\prime} \mathrm{s}<2.783\right)$. Simple effects analysis of the session $\times$ CS interaction revealed significant effects of the CS from session 3 onward $\left(F_{(1,15)}\right.$ 's $\left.>4.946\right)$. Significant effects of the session were observed for both the CS+ $\left(F_{(11,4)}=16.068\right.$, $P<0.01)$ and the CS- $\left(F_{(11,4)}=7.990, \quad P<0.05\right)$ magazine responding.

\section{Reactivation}

Figure $5 \mathrm{~B}$ shows the mean rate of magazine entry responding during the reactivation CS presentations in rats administered saline or MK-801 prior to the reactivation procedure. Inspection of this figure shows that rats administered both saline and MK-801 maintained a Pavlovian conditioned approach response to the $\mathrm{CS}+$ but not to the CS-. A mixed ANOVA with within factors of the CS (CS +, CS-) and a between-subjects factor of the drug (MK-801, saline) revealed a significant main effect of the CS $\left(F_{(1,14)}=25.175, P<0.001\right)$ and no significant main effect of the drug $\left(F_{(1,14)}=3.093, P=0.100\right)$. No significant interaction between the drug $\times$ CS was observed $\left(F_{(1,14)}=1.335\right.$, $P=0.267)$.

\section{Test performance}

Figure 5C shows the mean rate of magazine entry responding during the extinction test trial in rats administered saline or MK-801 prior to the reactivation procedure. Inspection of this figure shows that the groups of rats administered either saline or MK-801 maintained a Pavlovian conditioned approach response to the $\mathrm{CS}+$ but not to the CS-. A mixed ANOVA with within factors of the CS (CS + , CS-) and a between-subjects factor of drug (MK-801, saline) revealed a significant main effect of the CS $\left(F_{(1,14)}=\right.$ 13.045, $P<0.001)$ and no significant main effect of the drug $\left(F_{(1,14)}<1\right)$. No significant interaction between the drug $\times$ CS was observed $\left(F_{(1,14)}<1\right)$. Thus, MK-801 paired with the reactivation procedure did not abolish discrimination responding between the CS + and CS- in rats that had a 12-d Pavlovian conditioned approach training.

Because the reactivation procedure had no effect on discrimination performance in rats administered MK-801, no nonreactivation procedure was conducted in 12 -d trained rats.

This experiment demonstrates that a simple appetitive goaltracking procedure, previously demonstrated to be resistant to memory reconsolidation (Blaiss and Janak 2007), will undergo memory reconsolidation under distinct training conditions. It appears that an appetitive goal-tracking memory will undergo reconsolidation when rats are exposed to $6 \mathrm{~d}$ of discrimination training. In these rats, systemic treatment with the NMDA antagonist MK-801 prior to memory reactivation resulted in impaired discrimination due to the disruption of memory reconsolidation and maintained discrimination in saline-treated rats. However, a shorter training procedure of $3 \mathrm{~d}$ resulted in extinction learning in saline-treated rats, demonstrated by impaired discrimination at test. MK-801 treatment prevented the consolidation of extinction learning, whereby these rats continued to discriminate at test. Rats trained for $12 \mathrm{~d}$ continued to discriminate following a saline or MK-801 application, indicating that following extended training this goal-tracking memory was resistant to memory reconsolidation, at least using the current reactivation parameters.

\section{Experiment 2: Reactivation as a boundary constraint}

Experiment 1 showed that when keeping the parameters of memory reactivation constant (three nonreinforced exposures to each $\mathrm{CS})$, there is a limited parameter space for training (6 d) in order
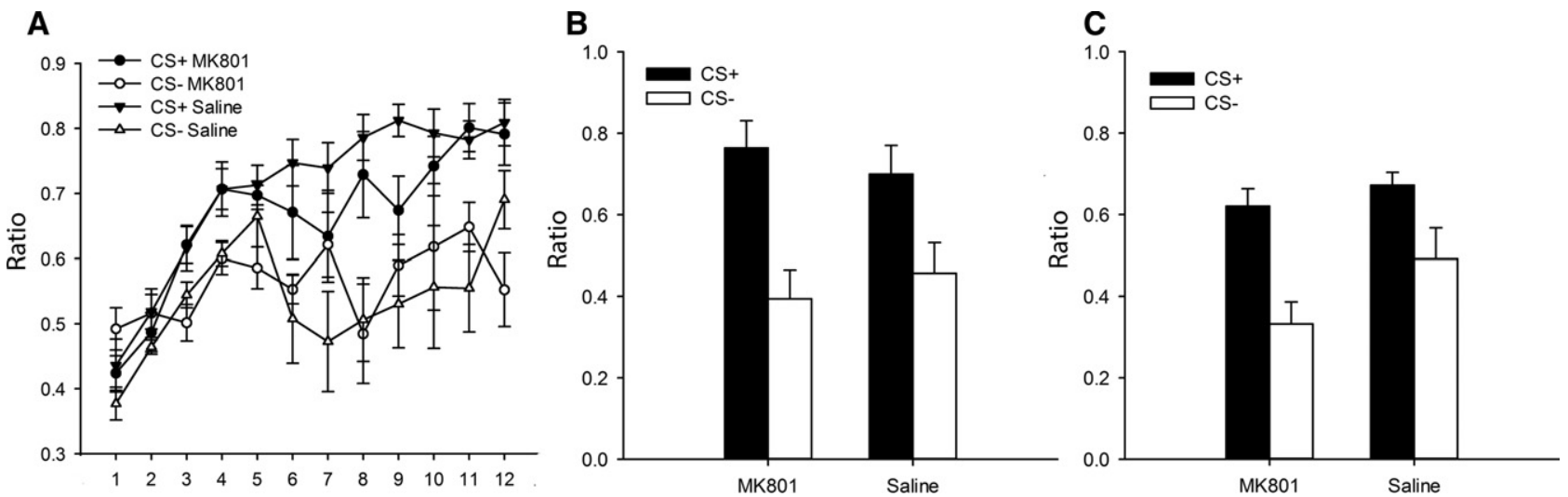

Figure 5. (A) Acquisition of individual auditory ( $C S+, C S-)$ discriminations across the 12-d training protocol (saline, $n=8 ; \mathrm{MK}-801, n=8)$. (B) Responding to three presentations each of nonreinforced CS + and CS - during the brief reactivation session. (C) Probe test responding to 10 presentations each of the CS + and CS - in extinction. Error bars represent \pm 1 SEM. All data are shown as ratios calculated as magazine responding during CS/ $(\mathrm{CS}+\mathrm{PreCS})$. 
to observe memory reconsolidation. This could be due either to a balance between the extents of training and nonreinforcement at memory reactivation (i.e., a ratio of nonreinforced CS presentations at reactivation to total number of reinforced presentations during training) or to an absolute training parameter constraint. In order to dissociate partially these alternative explanations, we investigated the impact of increasing the number of nonreinforced exposures of each CS at memory reactivation to six in the previously successful 6-day training condition. This shifts the balance of training to reactivation to that which is equivalent to the previous 3-day training condition, in which MK-801 impaired memory extinction rather than memory reconsolidation.

\section{Results}

One rat was excluded from the saline group for failing to acquire the discriminations during training (total, $n=15$; saline, $n=7$; MK-801, $n=8$ ).

\section{Acquisition of Pavlovian discriminations}

Figure $6 \mathrm{~A}$ shows the mean rate of magazine entry responding during the course of acquisition. Inspection of this figure shows that rats acquired a Pavlovian conditioned approach response to the $\mathrm{CS}+$ but not to the CS-. A within-subjects ANOVA with betweensubjects factors of the group (MK-801, saline) and within factors of the training session (1-6) and the CS $(\mathrm{CS}+, \mathrm{CS}-)$ revealed a significant main effect of the session $\left(F_{(5,65)}=19.081, P<0.001\right)$, a significant main effect of the CS $\left(F_{(1,13)}=26.148, P<0.001\right)$, and no significant main effect of the drug $\left(F_{(1,130}<1\right)$. A significant interaction between the session $\times$ CS was observed $\left(F_{(5,65)}=5.191, P<0.001\right)$. No further interactions were significant $\left(F^{\prime} \mathrm{s}<1.692\right)$. Simple effects analysis of the session $\times$ CS interaction revealed significant effects of the CS from session 4 onward $\left(F_{(1,13)}\right.$ 's $\left.>8.185\right)$. Significant effects of the session were observed for both the CS $+\left(F_{(5,9)}=16.523, P<0.001\right)$ and the CS $-\left(F_{(5,9)}=10.270, P<0.01\right)$ magazine responding.

\section{Reactivation}

Figure 6B shows the mean rate of magazine entry responding during the reactivation CS presentations in rats administered saline or MK-801 prior to the reactivation procedure. Inspection of this figure shows that rats administered both saline and MK-801 main- tained a Pavlovian conditioned approach response to the CS+ but not to the CS-. A mixed ANOVA with within factors of the CS $(\mathrm{CS}+, \mathrm{CS}-)$ and between-subjects factor of the drug (MK-801, saline) revealed a significant main effect of the CS $\left(F_{(1,13)}=6.661\right.$, $P<0.05)$ and no significant main effect of the drug $\left(F_{(1,13)}<1\right)$. No significant interaction between the drug $\times$ CS was observed $\left(F_{(1,13)}=1.679, P=0.218\right)$.

\section{Test performance}

Figure 6C shows the mean rate of magazine entry responding during the extinction test trial in rats administered saline or MK-801. Inspection of this figure shows that rats administered with saline or MK-801 maintained a Pavlovian conditioned approach response to the $\mathrm{CS}+$ but not to the $\mathrm{CS}-$. A mixed ANOVA with within factors of the CS (CS + , CS-) and a between-subjects factor of the drug (MK-801, saline) revealed a significant main effect of the CS $\left(F_{(1,13)}=5.170, P<0.05\right)$ and no significant main effect of the drug $\left(F_{(1,13)}=2.015, P=0.179\right)$. No significant interaction between the drug $\times$ CS was observed $\left(F_{(1,13)}<1\right)$. Thus, MK-801 did not abolish discrimination responding between the $\mathrm{CS}+$ and $\mathrm{CS}$ - in rats that underwent the double reactivation procedure.

This experiment demonstrates that the experimental conditions that engage the reconsolidation of a Pavlovian conditioned approach memory are more complex than a simple parametric calculation. It is neither true that there is a critical ratio of training reinforcement to reactivation nonreinforcement in order to trigger memory reconsolidation, nor is it the case that there is some critical level of initial memory acquisition that enables subsequent reconsolidation to take place. Thus, as demonstrated in Experiment 1B, following $6 \mathrm{~d}$ of training three nonreinforced presentations of each CS were sufficient to induce reconsolidation in a goal-tracking procedure. However, in Experiment $1 \mathrm{~B}$ in which rats were trained for $3 \mathrm{~d}$, the same number of nonreinforced reactivation trials was sufficient to induce extinction in rats administered saline, and MK-801 application resulted in impaired extinction consolidation. In Experiment 2, it was thought that if the number of reactivation trials were proportional to the 3-day training protocol, i.e., 3-d training, three nonreinforced presentations vs. 6 - $\mathrm{d}$ training, and six nonreinforced presentations, this procedure may evoke extinction as opposed to reconsolidation. However, this was not observed, and instead both salineand MK-801-administered rats biased responding to the CS +, suggesting that the boundary conditions are more complex than
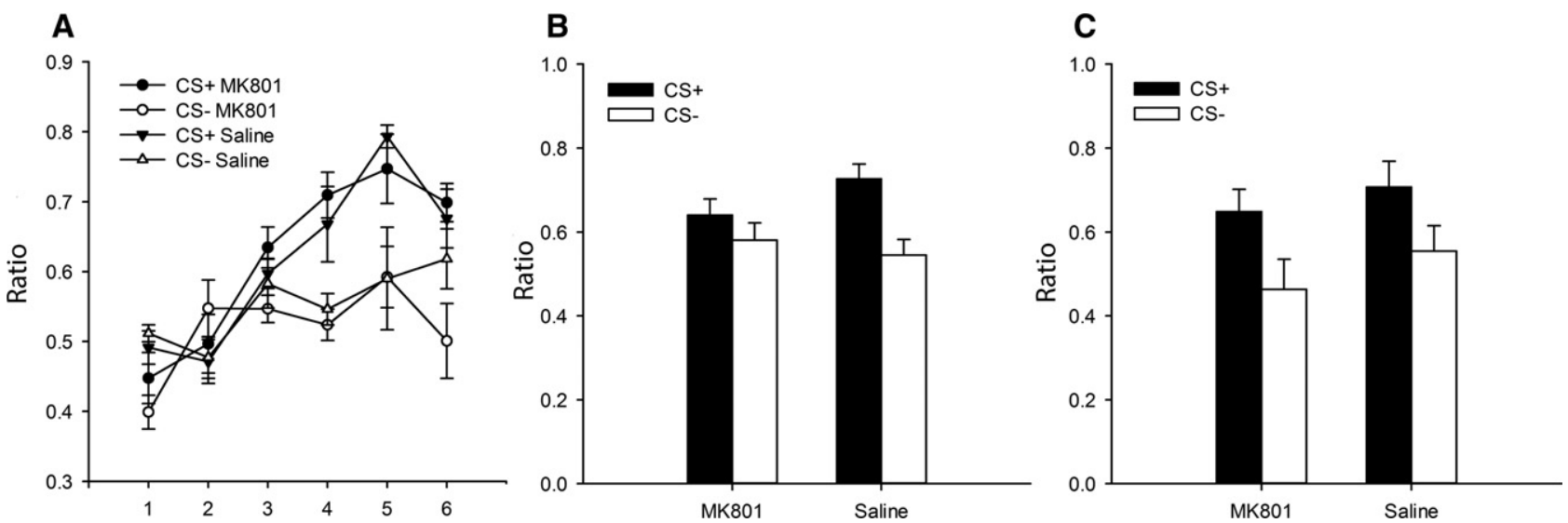

Figure 6. (A) Acquisition of individual auditory (CS,$+ \mathrm{CS}-$ ) discriminations across the 6-d training protocol (saline, $n=7 ; \mathrm{MK}-801, n=8)$. ( $B$ ) Responding to six presentations each of nonreinforced CS+ and CS- during the extended reactivation session. (C) Probe test responding to 10 presentations each of the CS + and CS- extinction. Error bars represent \pm 1 SEM. All data are shown as ratios calculated as magazine responding during CS/ $(\mathrm{CS}+$ PreCS). 
initially thought. Given that these results are not supportive of a purely parametric determination of reconsolidation, they may be more consistent with hypotheses that place the informational value of the reactivation session as being important for triggering reconsolidation.

\section{Discussion}

These experiments demonstrate that under discrete conditions an appetitive Pavlovian goal-tracking memory undergoes reconsolidation, and this can be disrupted through a prereactivation application of the NMDA subtype of glutamate receptor (NMDAR) antagonist MK-801, resulting in amnesia. However, under different behavioral conditions MK-801 can impair extinction to result in the opposite outcome of maintaining behavioral responding at test or has no discernible impact upon subsequent behavior. These results point toward discrete conditions within which memory reconsolidation is engaged. These conditions are not simply defined in terms of ratios between the extent of training and nonreinforced stimulus exposure at memory reactivation.

In the present study, we were able to demonstrate that following $6 \mathrm{~d}$ of training Pavlovian goal-tracking memories underwent reconsolidation following a short reactivation procedure. Application of MK-801 resulted in impaired discrimination, whereas saline-treated rats continued to discriminate between the CS + and CS-. This indicated that NMDAR antagonism is capable of disrupting memory reconsolidation following memory reactivation. In contrast, reactivation of a memory from $3 \mathrm{~d}$ of training resulted in extinction in saline controls, whereby salinetreated rats did not discriminate between the CS + and CS- at test. MK-801-treated rats continued to discriminate at test, indicating that the MK-801 application resulted in impaired extinction consolidation. This observation was dependent upon the reactivation/extinction procedure, as rats that did not undergo memory reactivation continued to discriminate between cues at test. The NMDAR-dependence of Pavlovian appetitive memory extinction has previously been demonstrated, whereby MK-801 acts to disrupt extinction learning consolidation (Bevins and Bardo 1999; Delamater 2004; Dalley et al. 2005).

Typically, in studies of Pavlovian memory reconsolidation, memory reactivation is achieved by exposure to the CS (whether it is discrete or contextual) in the absence of the US. Operationally, therefore, memory reactivation actually consists of a brief extinction training session. The behavioral impact of extinction training is to diminish subsequent memory expression (Rescorla 2001; Bouton 2004), which under most circumstances results from new inhibitory learning (Rescorla 2001; Kehoe and White 2002; Bouton 2004; Delamater 2004). Whether a memory will undergo reconsolidation or extinction following the reactivation procedure provides a fundamental boundary condition. In fact, if the reactivation procedure induces extinction learning, amnestic treatment would impair such new inhibitory learning, thereby resulting in a preserved memory expression. This effect is observed under conditions that favor extinction over reconsolidation, such as those in Experiment $1 \mathrm{a}$, whereby $3 \mathrm{~d}$ of training did not generate a suitably robust memory.

The competition between reactivation/reconsolidation and extinction and their opposing effects on subsequent memory expression has been conceptualized within a "trace dominance" framework (Eisenberg et al. 2003). That is, the memory trace that is dominantly activated by the reactivation/extinction session is the one that is impaired by amnestic treatment. This may be due to there being competition between the CS-US memory and the extinction of CS-no US memory for "cellular plasticity resources" when the memory is retrieved. Therefore, if extinction is dominantly engaged, amnestic treatment will impair extinction to preserve the subsequent memory expression. In contrast, if reconsolidation of the original excitatory trace is dominantly activated, then memory restabilization will be disrupted, leading to diminished memory expression. We have previously demonstrated such a trace dominance using MK-801 in Pavlovian fear conditioning, whereby reconsolidation was engaged by a brief unreinforced CS exposure, and extinction by more prolonged extinction training (Lee et al. 2006b). The present results are conceptually similar, though they are revealed through varying the extent of training rather than reactivation/extinction, as was originally demonstrated by Eisenberg et al. (2003) in a conditioned taste-aversion setting. It should be noted that the central locus of effect of systemically administered MK-801 has not been delineated in our present or previous studies. In addition, there is no reason to assume that the central locus of effect is the same for the reconsolidation and extinction effects. Nevertheless, it appears that the parametric factors that determine the balance between reactivation and extinction are the strength of training and the extent of unreinforced stimulus exposure at memory retrieval.

Despite the growing evidence for a competition between reconsolidation and extinction (Eisenberg et al. 2003; Pedreira and Maldonado 2003; Suzuki et al. 2004; Lee et al. 2006b; de la Fuente et al. 2011), it has also been reported that even under conditions that induced appreciable extinction, intra-basolateral amygdala (BLA) infusions of anisomycin-impaired reconsolidation diminish conditioned freezing (Duvarci et al. 2006). Therefore, when reconsolidation is impaired to disrupt performance in the present 6-day training study, this might occur in parallel with a disruption of extinction processes. However, it cannot be the case similarly that reconsolidation is engaged and disrupted in parallel with extinction in the 3-day training condition, as performance was not impaired. Thus extinction does appear to take place in the absence of reconsolidation. Nevertheless, the present results do lend further support to the interpretation that extinction per se does not provide a boundary condition on reconsolidation. When the duration of memory reactivation was doubled in the 6-day training condition, there was no behavioral effect of MK-801. Therefore, neither reconsolidation nor extinction was disrupted by MK-801, and so increasing the duration of stimulus exposure at memory reactivation reveals a boundary condition that cannot be explained by trace dominance. It remains to be demonstrated that further prolonged stimulus exposure engages memory extinction that is disrupted by MK-801. What is clear, however, is that there is no simple linear parametric calculation that determines the amnestic effect of MK-801. The ratio of reactivation to training was the same in the 6-day training double reactivation condition as it was in the 3-day training normal reactivation condition, yet only in the latter was extinction engaged and disrupted by MK-801.

Previously, Blaiss and Janak (2007) reported that an appetitive Pavlovian conditioned approach memory was not affected by the protein synthesis inhibitor anisomycin administered systemically at the time of memory reactivation; this typically impairs memory reconsolidation and results in amnesia (Rudy et al. 2006). This failure to observe reconsolidation of the underlying memory occurred regardless of changes to the extent of training and stimulus reexposure at memory reactivation. There is no clear reason for the failure of the study by Blaiss and Janak (2007) to reveal evidence for memory reconsolidation in a goaltracking setting. One notable experimental difference in the current study was the amnestic treatment used. While sensitivity to postreactivation protein synthesis inhibition is the current prototypical demonstration of memory reconsolidation, MK-801 (an antagonist at NMDAR) has been used extensively in memory 
reconsolidation studies (Przybyslawski and Sara 1997; Lee et al. 2006a), including in appetitive settings (Kelley et al. 2007; Sadler et al. 2007; Lee and Everitt 2008a,b,c; Milton et al. 2008a, 2012). In all of these studies MK- 801 was administered shortly prior to memory reactivation, unlike the postreactivation administration of protein synthesis inhibitors. However, Lee and Everitt (2008b) previously demonstrated that the administration of MK-801 prior to, but not following, response contingent memory reactivation reduced subsequent cue-induced sucrose seeking. Postreactivation MK-801 administration also failed to impair appetitive memory reconsolidation in the maintenance of an acquired instrumental response with conditioned reinforcement (Lee and Everitt 2008b). These studies are indicative of a critical NMDAR-dependent signaling period that lasts for only a short period after the reactivation session, and hence is more sensitive to the prereactivation administration of NMDAR antagonists. Moreover, the amnestic effect of MK-801 was critically dependent upon the reactivation session, strongly indicating that it results from a disruption of memory reconsolidation. Therefore, the selection of amnestic agent is unlikely to account for the difference in observations between the current study and that of Blaiss and Janak (2007). It remains to be demonstrated, however, that protein synthesis inhibition can replicate the amnestic effect of MK-801 in the current setting.

While the behavioral protocols between the current study and that of Blaiss and Janak (2007) do differ subtly, the latter did vary both the extent of training (from 30 to 105 CS-US pairings), keeping the reactivation constant (five CS presentations), and using 60 CS-US pairings at training compared a single CS presentation to five CS presentations. They observed no difference between treated and control rats, indicating that neither reconsolidation nor extinction were disrupted. Therefore, it was not due to trace dominance that Blaiss and Janak did not disrupt reconsolidation. A simple parametric comparison reveals that the present optimal training condition ( $6 \mathrm{~d}$ of $10 \mathrm{CS}-\mathrm{US}$ pairings per session) is equivalent to the $4 \mathrm{~d}$ of $15 \mathrm{CS}-\mathrm{US}$ pairings employed by Blaiss and Janak (2007). However, in our study, only three CS reexposures (and not six) were successful in reactivating the memory. This might suggest that the five CS presentations used by Blaiss and Janak (2007) was too extensive. Moreover, Suzuki et al. (2004) have previously shown that there is a minimum amount of stimulus reexposure that is necessary to destabilize a contextual fear memory successfully. Therefore, it is possible that by chance Blaiss and Janak (2007) missed the parameter space necessary to observe reconsolidation. If this is the case, it serves further to reinforce the restricted area of such a parameter space.

The most extensive training parameters used by Blaiss and Janak (2007) are similar to those employed in the 12-day training condition in the present study, which revealed some evidence for a further boundary condition on memory reconsolidation exerted by the strength of training. The stronger memory formed from $12 \mathrm{~d}$ of Pavlovian training was not disrupted by MK-801 injection prior to a brief memory reactivation. It remains unclear whether this reflects an absolute constraint on memory reconsolidation (i.e., that 12-day trained memories do not undergo reconsolidation under any reactivation conditions). An alternative hypothesis is that a more extended stimulus exposure would have successfully reactivated the 12-day trained memory and stimulated its reconsolidation. In contextual fear conditioning, a more strongly conditioned memory required more prolonged context reexposure in order to reactivate the memory and render it susceptible to the amnestic effects of protein synthesis inhibition (Suzuki et al. 2004). What may be concluded from both the present results and those of Suzuki et al. (2004) is that stronger memories are not as easily destabilized by a brief stimulus reexposure as are more weakly conditioned memories. However, the current 12-d training setting is semiconfounded by the increased age of this memory. Memory age has also been demonstrated to exert a boundary condition on memory reconsolidation in a contextual fear paradigm in mice (Suzuki et al. 2004). 8-week old memories were more resistant to memory reactivation than 1 - and 3-week old memories, consistent with observations in both a rodent inhibitory avoidance setting (Milekic and Alberini 2002) and fear conditioning in Medaka fish (Eisenberg and Dudai 2004). Nevertheless, increasing the duration of stimulus reexposure was again able successfully to reactivate the older memories (Suzuki et al. 2004). The age of the 12-day memory in the current study may be consistent with a memory age boundary condition, although it is not clear whether the critical parameter is the time elapsed from the start or end of training, and it remains to be determined whether some other magnitude or method of memory retrieval would have successfully destabilized the goal-tracking memory.

\section{Conclusions}

This study demonstrates two novel observations: (1) That a Pavlovian goal-tracking memory, previously thought to be resistant to destabilization, will undergo memory reconsolidation under discrete conditions that favor reconsolidation as opposed to extinction, and (2) that the boundary conditions that exist for (appetitive) memory reconsolidation are much more complex than simple parametric calculations; a memory that would previously undergo destabilization following one protocol of reactivation will not undergo reconsolidation or extinction when the reactivation is extended.

\section{Materials and Methods}

\section{Experiment 1}

Subjects

The subjects were 104 experimentally naive adult male Lister Hooded rats (supplied by Harlan OLAC, UK), weighing 250$300 \mathrm{~g}$. They were housed in groups of four in holding rooms maintained at $21^{\circ} \mathrm{C}$ on a standard light cycle $(12 \mathrm{~h}$ light/dark cycle; lights on at 7 a.m.). Food was restricted to $15 \mathrm{~g} /$ day; water was available ad libitum throughout the experiment. Training and testing were conducted between $9 \mathrm{a} . \mathrm{m}$. and $12 \mathrm{p} . \mathrm{m}$. All procedures were conducted in accordance with the United Kingdom 1986 Animals (Scientific Procedures) Act (Project License PPL 40/3205).

\section{Drugs}

The rats were administered (+)-5-methyl-10,11-dihydro-5Hdibenzo[ $a, d]$ cyclohepten-5,10-imine maleate (MK-801, Sigma) dissolved in saline $(0.1 \mathrm{mg} / \mathrm{mL})$ at a dose of $0.1 \mathrm{mg} / \mathrm{kg}$ by intraperitoneal (i.p.) injection $30 \mathrm{~min}$ prior to the reactivation procedure. Saline served as the vehicle control.

\section{Apparatus}

Pavlovian conditioned approach training was performed in eight identical, standard operant chambers $(30 \mathrm{~cm}$ wide, $21 \mathrm{~cm}$ high, and $24 \mathrm{~cm}$ deep, and supplied by Med Associates) contained in sound-attenuating boxes and arranged in a four-by-two array in a testing room. Each chamber consisted of three walls and a ceiling, with the door serving as the fourth wall. The ceiling, door, and back wall were made from clear Perspex and the left and right walls were made from stainless steel. The floor of each chamber was constructed of 19 stainless steel rods $(4.8 \mathrm{~mm}$ in diameter, spaced $16 \mathrm{~mm}$ apart). Each chamber was illuminated by a $3 \mathrm{~W}$ house light located at the top center of the left wall. The right walls of the chambers were fitted with a recessed magazine into which food or sucrose pellets ( $45 \mathrm{mg}$; P.J. Noyes) were delivered. 
Two flat-panel, retractable levers were located to the left and right of the magazine; these remained retracted throughout the experiment. Above each lever was a 2 -cm diameter panel light. The magazine entries were detected by an infrared sensor. Auditory stimuli consisted of a $2-\mathrm{kHz}$ pure tone delivered from a speaker located in the left wall and a $2-\mathrm{Hz}$ train of clicks $(80 \mathrm{~dB})$ generated by a relay clicker, also located in the left wall. A computer equipped with MED-PC software (version IV; Med Associates Inc.) controlled the chambers and recorded the data.

\section{Procedure}

Behavioral training. Pavlovian training was carried out over (a) 3, (b) 6 , or (c) $12 \mathrm{~d}$ (one session per day) during which two discriminable auditory stimuli (click or tone) were presented 10 times each session for $30 \mathrm{sec}$. One stimulus (the $\mathrm{CS}+$, counterbalanced across the rats) was reinforced with the delivery of three sucrose pellets at the end of the presentation, whereas the CS- was never reinforced. Each stimulus presentation was separated by a 60 -sec period, separated into a 30 -sec intertrial interval (ITI) and a 30-sec PreCS period, in which no cues were presented. Discrimination performance was measured by ratios of magazine responding during the CS presentation and the preceding period as calculated by the equation CS/(CS + PreCS).

Reactivation. In the reactivation groups, MK-801 (0.1 mg/kg i.p.) or vehicle (saline) was administered $30 \mathrm{~min}$ prior to the reactivation procedure. The Pavlovian CS-sucrose memory was reactivated in a short extinction session where three presentations each of the CS+ and CS- were presented without being rewarded. Each stimulus presentation was separated by a 60 -sec period, separated into a 30-sec ITI and a 30-sec PreCS period, in which no cues were presented. In nonreactivation control conditions, the rats were administered MK-801 $(0.1 \mathrm{mg} / \mathrm{kg}$ i.p.) or vehicle (saline) in the holding room, but did not undergo the reactivation procedure.

Test. At test, $24 \mathrm{~h}$ following reactivation (or nonreactivation in control rats), goal-tracking activity was measured by magazine entry behaviors during nonreinforced CS + and CSpresentations, and thus the clicks or tones were presented 10 times for $30 \mathrm{sec}$, each trial separated by a 60 -sec stimulus-free period, separated into a $30-$ sec ITI and a $30-$ sec PreCS period.

\section{Experiment 2}

\section{Subjects}

The subjects were 16 experimentally naive adult male Lister Hooded rats (supplied by Harlan OLAC, UK), weighing 250$300 \mathrm{~g}$. They were housed, trained, and tested as described in Experiment 1.

\section{Drugs}

The rats were administered MK-801 (Sigma) dissolved in saline $(0.1 \mathrm{mg} / \mathrm{mL})$ at a dose of $0.1 \mathrm{mg} / \mathrm{kg}$ by i.p. injection $30 \mathrm{~min}$ prior to the reactivation procedure. Saline served as the vehicle control.

\section{Apparatus}

Pavlovian conditioned approach training was performed in the same apparatus as in Experiment 1.

\section{Procedure}

Behavioral training and test. Pavlovian training and testing was carried out over $6 \mathrm{~d}$ (one session per day) as described previously for Experiment 1.
Reactivation. In the reactivation groups, MK-801 (0.1 mg/kg i.p.) or vehicle (saline) was administered $30 \mathrm{~min}$ prior to the reactivation procedure. The Pavlovian CS-sucrose memory was reactivated in a short extinction session where six presentations each of the CS+ and CS- were presented without being rewarded. Each stimulus presentation was separated by a 60 -sec period, separated into a $30-\mathrm{sec}$ ITI and a $30 \mathrm{sec}$ PreCS period, in which no cues were presented. In nonreactivation control conditions, the rats were administered MK-801 ( $0.1 \mathrm{mg} / \mathrm{kg}$ i.p.) or vehicle (saline) in the holding room, but did not undergo the reactivation procedure.

\section{Acknowledgments}

This study was supported by funds from the Leverhulme Trust Grant F/00 094/BK awarded to J.L.C.L. We thank David Barber for his assistance with data collection.

\section{References}

Alberini CM. 2005. Mechanisms of memory stabilization: Are consolidation and reconsolidation similar or distinct processes? Trends Neurosci 28: 51-56.

Bevins RA, Bardo MT. 1999. Conditioned increase in place preference by access to novel objects: Antagonism by MK-801. Behav Brain Res 99: $53-60$.

Blaiss CA, Janak PH. 2007. Post-training, but not post-reactivation, administration of amphetamine and anisomycin modulates Pavlovian conditioned approach. Neurobiol Learn Mem 87: 644-658.

Bouton ME. 2004. Context and behavioral processes in extinction. Learn Mem 11: 485-494.

Dalley JW, Laane K, Theobald DE, Armstrong HC, Corlett PR, Chudasama Y, Robbins TW. 2005. Time-limited modulation of appetitive Pavlovian memory by D1 and NMDA receptors in the nucleus accumbens. Proc Natl Acad Sci 102: 6189-6194.

de la Fuente V, Freudenthal R, Romano A. 2011. Reconsolidation or extinction: Transcription factor switch in the determination of memory course after retrieval. J Neurosci 31: 5562-5573.

Delamater AR. 2004. Experimental extinction in Pavlovian conditioning: Behavioural and neuroscience perspectives. Q J Exp Psychol B 57: 97-132.

Diergaarde L, Schoffelmeer AN, De Vries TJ. 2006. Beta-adrenoceptor mediated inhibition of long-term reward-related memory reconsolidation. Behav Brain Res 170: 333-336.

Duvarci S, Mamou CB, Nader K. 2006. Extinction is not a sufficient condition to prevent fear memories from undergoing reconsolidation in the basolateral amygdala. Eur J Neurosci 24: 249-260.

Eisenberg M, Dudai Y. 2004. Reconsolidation of fresh, remote, and extinguished fear memory in Medaka: Old fears don't die. Eur J Neurosci 20: $3397-3403$.

Eisenberg M, Kobilo T, Berman DE, Dudai Y. 2003. Stability of retrieved memory: Inverse correlation with trace dominance. Science 301: 1102-1104.

Finnie PS, Nader K. 2012. The role of metaplasticity mechanisms in regulating memory destabilization and reconsolidation. Neurosci Biobehav Rev 36: 1667-1707.

Hardt O, Einarsson EO, Nader K. 2010. A bridge over troubled water: Reconsolidation as a link between cognitive and neuroscientific memory research traditions. Annu Rev Psychol 61: 141-167.

Hernandez PJ, Kelley AE. 2004. Long-term memory for instrumental responses does not undergo protein synthesis-dependent reconsolidation upon retrieval. Learn Mem 11: 748-754.

Inda MC, Muravieva EV, Alberini CM. 2011. Memory retrieval and the passage of time: From reconsolidation and strengthening to extinction. J Neurosci 31: 1635-1643.

Kehoe EJ, White NE. 2002. Extinction revisited: Similarities between extinction and reductions in US intensity in classical conditioning of the rabbit's nictitating membrane response. Anim Learn Behav 30: 96-111.

Kelley JB, Anderson KL, Itzhak Y. 2007. Long-term memory of cocaine-associated context: Disruption and reinstatement. Neuroreport 18: $777-780$.

Konorski J. 1967. Some new ideas concerning the physiological mechanisms of perception. Acta Biol Exp (Warsz) 27: 147-161.

Lee JL. 2008. Memory reconsolidation mediates the strengthening of memories by additional learning. Nat Neurosci 11: 1264-1266.

Lee JL. 2009. Reconsolidation: Maintaining memory relevance. Trends Neurosci 32: 413-420. 
Lee JL, Everitt BJ. 2008a. Appetitive memory reconsolidation depends upon NMDA receptor-mediated neurotransmission. Neurobiol Learn Mem 90: $147-154$.

Lee JL, Everitt BJ. 2008b. Reactivation-dependent amnesia for appetitive memories is determined by the contingency of stimulus presentation. Learn Mem 15: 390-393.

Lee JL, Everitt BJ. 2008c. Reactivation-dependent amnesia in Pavlovian approach and instrumental transfer. Learn Mem 15: 597-602.

Lee JL, Di Ciano P, Thomas KL, Everitt BJ. 2005. Disrupting reconsolidation of drug memories reduces cocaine-seeking behavior. Neuron 47: 795-801.

Lee JL, Milton AL, Everitt BJ. 2006a. Cue-induced cocaine seeking and relapse are reduced by disruption of drug memory reconsolidation. J Neurosci 26: 5881-5887.

Lee JL, Milton AL, Everitt BJ. 2006b. Reconsolidation and extinction of conditioned fear: Inhibition and potentiation. J Neurosci 26: 10051-10056.

Milekic MH, Alberini CM. 2002. Temporally graded requirement for protein synthesis following memory reactivation. Neuron 36: 521-525.

Milton AL, Lee JL, Butler VJ, Gardner R, Everitt BJ. 2008a. Intra-amygdala and systemic antagonism of NMDA receptors prevents the reconsolidation of drug-associated memory and impairs subsequently both novel and previously acquired drug-seeking behaviors. J Neurosci 28: 8230-8237.

Milton AL, Lee JL, Everitt BJ. 2008b. Reconsolidation of appetitive memories for both natural and drug reinforcement is dependent on beta-adrenergic receptors. Learn Mem 15: 88-92.

Milton AL, Schramm MJ, Wawrzynski JR, Gore F, Oikonomou-Mpegeti F, Wang NQ, Samuel D, Economidou D, Everitt BJ. 2012. Antagonism at NMDA receptors, but not $\beta$-adrenergic receptors, disrupts the reconsolidation of pavlovian conditioned approach and instrumental transfer for ethanol-associated conditioned stimuli.

Psychopharmacology 219: 751-761.
Nader K, Einarsson EO. 2010. Memory reconsolidation: An update. Ann N Y Acad Sci 1191: 27-41.

Nader K, Hardt O. 2009. A single standard for memory: The case for reconsolidation. Nat Rev Neurosci 10: 224-234.

Pedreira ME, Maldonado H. 2003. Protein synthesis subserves reconsolidation or extinction depending on reminder duration. Neuron 38: $863-869$

Przybyslawski J, Sara SJ. 1997. Reconsolidation of memory after its reactivation. Behav Brain Res 84: $241-246$.

Rescorla RA. 2001. Are associative changes in acquisition and extinction negatively accelerated? J Exp Psychol Anim Behav Process 27: 307-315.

Robbins TW. 1990. In vivo neurochemical techniques in the analysis of behaviour. J Psychopharmacol 4: 244

Rudy JW, Biedenkapp JC, Moineau J, Bolding K. 2006. Anisomycin and the reconsolidation hypothesis. Learn Mem 13: 1-3.

Sadler R, Herzig V, Schimdt WJ. 2007. Repeated treatment with the NMDA antagonist MK-801 disrupts reconsolidation of memory for amphetamine-conditioned place preference. Behav Pharmacol 18: 699-703.

Suzuki A, Josselyn SA, Frankland PW, Masushige S, Silva AJ, Kida S. 2004. Memory reconsolidation and extinction have distinct temporal and biochemical signatures. J Neurosci 24: 4787-4795.

Tronson NC, Taylor JR. 2007. Molecular mechanisms of memory reconsolidation. Nat Rev Neurosci 8: 262-275.

Wouda JA, Diergaarde L, Riga D, van Mourik Y, Schoffelmeer AN, De Vries TJ. 2010. Disruption of long-term alcohol-related memory reconsolidation: Role of beta-adrenoceptors and NMDA receptors. Front Behav Neurosci 4: 179.

Received June 14, 2012; accepted in revised form November 5, 2012. 


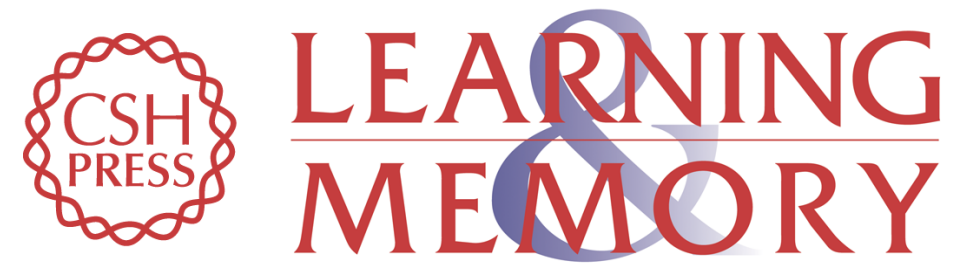

\section{Appetitive Pavlovian goal-tracking memories reconsolidate only under specific conditions}

Amy C. Reichelt and Jonathan L.C. Lee

Learn. Mem. 2013, 20:

Access the most recent version at doi:10.1101//m.027482.112

References This article cites 41 articles, 14 of which can be accessed free at: http://learnmem.cshlp.org/content/20/1/51.full.html\#ref-list-1

License

Email Alerting Receive free email alerts when new articles cite this article - sign up in the box at the Service top right corner of the article or click here. 